\title{
A RESULT OF COMMUTATIVITY OF RINGS
}

\author{
VISHNU GUPTA \\ Department of Mathematics \\ M.D. University, P.G. Regional Centre \\ Rewari (Haryana) INDIA
}

(Received December 11, 1990 and in revised form September 9, 1991)

Abstract. In this paper we prove the following:

THEOREM. Let $\boldsymbol{n}>1$ and $\boldsymbol{m}$ be fixed relatively prime positive integers and $\boldsymbol{k}$ is any non-negative integer. If $R$ is a ring with unity 1 satisfying $x^{k}\left[x^{n}, y\right]=\left[x, y^{m}\right]$ for all $x, y \in R$ then $R$ is commutative.

Key Words and Phrases: Commutator ideal, nilpotent elements.

1991 Mathematics Subject Classification: $16 \mathrm{~A} 70$.

\section{INTRODUCTION.}

Psomopoulos [12] proved that if $R$ is a ring with unity satisfying the properties that for each $x, y \in R$,

(i) $x^{k}\left[x^{n}, y\right]=\left[x, y^{m}\right]$

(ii) $\quad(x y)^{n}=x^{n} y^{n}$

(iii) $\quad(x y)^{k}=x^{k} y^{k}$

where $n>1$ and $m$ are fixed relatively prime positive integers and $k$ is any non-negative integer, then $R$ is commutative. In this paper we prove the theorem stated in the abstract which improve above theorem of Psomopolous [12] where conditions (ii) and (iii) are superfluous.

Throughout, $R$ will denote an associative ring with unit 1 . We use the following notations.

$Z(R)$, the center of $R$.

$[x, y]=x y-y x$

$C(R)$, the commutator ideal of $R$.

$N(R)$, the set of all nilpotent elements of $R$.

$D(R)$, the set of all zero divisors in $R$.

\section{MAIN RESULTS.}

We state our main result as follows.

MAIN THEOREM. Let $n>1$ and $m$ be fixed relatively prime positive integers and $k$ is any nonnegative integer. If $R$ is a ring with unity 1 satisfying

$$
x^{k}\left[x^{n}, y\right]=\left[x, y^{m}\right] \text { for all } x, y \in R
$$

then $R$ is commutative.

We begin with the following lemmas which will be used in proving our main theorem. 
LEMMA 1 ([2], Theorem 1). Let $R$ be a ring satisfying an identity $q(X)=0$, where $q(X)$ is a polynomial identity in non-commuting in-determinates, its coefficient being integers with highest common factor one. If there exists no prime $p$ for which the ring of $2 \times 2$ matrices over $G F(p)$ satisfies $q(X)=0$, then $R$ has a nil commutator ideal and the nilpotent elements of $R$ form an ideal.

LEMMA 2 ([8], p. 221). If $x, y \in R$ and $[x, y]$ commute with $x$, then $\left[x^{n}, y\right]-n x^{n-1}[x, y]$ for all positive integer $n$.

LEMMA 3 ([9]). Let $R$ be a ring with unity and let $f: R \rightarrow R$ be a function such that $f(x+1)-f(x)$ for all $x \in R$. If for some positive integer $n, x^{n} f(x)-0$ for all $x$ in $R$, then necessarily $f(x)=0$.

LEMMA 4. If $R$ is a ring satisfying $\left(^{*}\right)$ in the hypothesis of the main theorem then

$$
C(R) \subseteq N(R) \subseteq Z(R)
$$

PROOF. By Lemma 3 of [12] we have $N(R) \subseteq Z(R)$ when $R$ satisfies $x^{k}\left[x^{n}, y\right]-\left[x, y^{\prime \prime}\right]$ for all $x, y \in R$. This is a polynomial identity with coprime integral coefficients. But if we consider (i) $x=e_{22}$ and $y=e_{21}$, if $n>1, m>1$ and (ii) $x-e_{21}$ and $y=e_{22}$ if $n>1$ and $m=1$, we find that no ring of $2 \times 2$ matrices over $G F(p), p$ a prime, satisfies this identity. Hence by Lemma $1, C(R)$ is a nil ideal and thus

$$
C(R) \subseteq N(R) \subseteq Z(R) \text {. }
$$

PROOF OF MAIN THEOREM. By Lemma 4, we have

$$
C(R) \subseteq N(R) \subseteq Z(R)
$$

Thus all commutators are central. Moreover, we know that $R$ is isomorphic to a subdirect sum of subdirectly irreducible rings $\boldsymbol{R}_{\mathrm{a}}$ each of which a homomorphic image of $R$ satisfies the hypotheses of the theorem. Thus we can assume that $R$ is subdirectly irreducible ring. Hence $I$, the intersection of all non-zero ideals is non-zero.

CASE 1. Let $n>1$ and $m>1$.

By using Lemma 2, we write $\left(^{*}\right)$ as

$$
n x^{n+k-1}[x, y]-\left[x, y^{m}\right] \text { for all } x, y \in R \text {. }
$$

Let $c=2^{n+k}-2>0$, then

$$
\begin{aligned}
n c x^{n+k-1}[x, y] & =n\left\{2^{n+k} x^{n+k-1}[x, y]-2 x^{n+k-1}[x, y]\right\} \\
& =n 2^{n+k} x^{n+k-1}[x, y]-2 n x^{n+k-1}[x, y] \\
& =n(2 x)^{n+k-1}[2 x, y]-2\left[x, y^{m}\right] \\
& =\left[2 x, y^{m}\right]-2\left[x, y^{m}\right]=0 .
\end{aligned}
$$

Hence $n c x^{n+k-1}[x, y]=0$ for all $x, y \in R$. Now replace $x$ by $x+1$ and by using Lemma 3 , we get

$$
n c[x, y]=0 \text {. }
$$

All cummutators are central and hence by Lemma 2

$$
\left[x^{m c}, y\right]=n c x^{m c-1}[x, y]=0 \text {. }
$$

Thus $x^{n c} \in Z(R)$ for all $x \in R$. We replace $y$ by $y^{m}$ in (2.1) to get

$$
n x^{n+k-1}\left[x, y^{m}\right]=\left[x,\left(y^{m}\right)^{m}\right] \text {. }
$$


Thus

and

$$
\begin{aligned}
n x^{n+k-1}\left[x, y^{m}\right] & =n\left[x, y^{m}\right] x^{n+k-1} \\
& =n m y^{m-1}[x, y] x^{n+k-1} \\
& =n m y^{m-1} x^{n+k-1}[x, y] \\
& =m y^{m-1}\left[x, y^{m}\right]
\end{aligned}
$$

$$
\left[x,\left(y^{m}\right)^{m}\right]=m\left(y^{m}\right)^{m-1}\left[x, y^{m}\right]=m y^{m-1} y^{(m-1)^{2}}\left[x, y^{m}\right]
$$

Thus by using (2.5) and (2.6), we can write (2.4) as

$$
\begin{gathered}
m y^{m-1}\left[x, y^{m}\right]=m y^{m-1} y^{(m-1)^{2}}\left[x, y^{m}\right] \\
m y^{m-1}\left(1-y^{(m-1)^{2}}\right)\left[x, y^{m}\right]=0
\end{gathered}
$$

Hence

$$
m y^{m-1}\left(1-y^{m c(m-1)^{2}}\right)\left[x, y^{m}\right]=0 .
$$

We claim that

$$
D(R) \subseteq Z(R) .
$$

Let $a \in D(R)$ then

$$
a^{n c(m-1)^{2}} \in Z(R) \cap D(R) \text { and } I a^{m c(m-1)^{2}}=0
$$

By (2.7), we get

$$
m a^{m-1}\left(1-a^{m c(m-1)^{2}}\right)\left[x, a^{m}\right]=0 .
$$

Thus

$$
\left(1-a^{n c(m-1)^{7}}\right) m a^{m-1}\left[x, a^{m}\right]=0 \text {. }
$$

If $m a^{m-1}\left[x, a^{m}\right] \neq 0$, then

$$
1-a^{n c(m-1)^{2}} \in D(R)
$$

Hence $I\left(1-a^{n c(m-1)^{2}}\right)=0$ and $I=0$. This is contradiction. Now we have

$$
m a^{m-1}\left[x, a^{m}\right]=0 .
$$

Thus

$$
\begin{aligned}
n^{2} x^{n+k-1} x^{n+k-1}[x, a] & =n x^{n+k-1}\left[x, a^{m}\right] \\
& =\left[x,\left(a^{m}\right)^{m}\right] \\
& =m\left(a^{m}\right)^{m-1}\left[x, a^{m}\right] \\
& =a^{(m-1)^{2}} m a^{m-1}\left[x, a^{m}\right]=0 .
\end{aligned}
$$

Replacing $x$ by $x+1$ in (2.10) and using Lemma 3 we get

$$
n^{2}[x, a]=0 \text {. }
$$

By using Lemma 2, we can write $\left({ }^{*}\right)$ as

$$
x^{k}\left[x^{n}, y\right]=m y^{m-1}[x, y] .
$$

Let $d=2^{m}-2>0$. Then 


$$
\begin{aligned}
m d y^{m-1}[x, y] & =m 2^{m} y^{m-1}[x, y]-2 y^{m-1}[x, y] \\
& =m(2 y)^{m-1}[x, 2 y]-2 m y^{m-1}[x, y] \\
& =x^{k}\left[x^{n}, 2 y\right]-2 x^{k}\left[x^{n}, y\right] \\
& =x^{k}\left[x^{n}, 2 y\right]-x^{k}\left[x^{n}, 2 y\right]=0 .
\end{aligned}
$$

Hence $m d y^{m-1}[x, y]=0$ for all $x, y \in R$. Now replacing $y$ by $y+1$ and by using Lemma 3 , we get

$$
m d[x, y]=0 \text {. }
$$

All commutators are central and hence by Lemma 2

$$
\left[x, y^{m d}\right]=m d y^{m d-1}[x, y]=0
$$

Thus $y^{m d} \in Z(R)$ for all $y \in R$. Now replacing $x$ by $x^{n}$ in (2.12), we get

$$
x^{n k}\left[\left(x^{n}\right)^{n}, y\right]=m y^{m-1}\left[x^{n}, y\right]
$$

Thus

$$
\begin{aligned}
x^{n k}\left[\left(x^{n}\right)^{n}, y\right] & =x^{m k} n\left(x^{n}\right)^{n-1}\left[x^{n}, y\right] \\
& =n x^{n k} x^{n-1} x^{(n-1)^{2}}\left[x^{n}, y\right] \\
& =n x^{n+k-1} x^{n-k} x^{(n-1)^{2}}\left[x^{n}, y\right] \\
& =n x^{n x^{n+k-1}} x^{(n-1)} x^{(n-1)^{2}}\left[x^{n}, y\right] \\
& =n x^{n+k-1} x^{(n-1)(n+k-1)}\left[x^{n}, y\right] \\
m y^{m-1}\left[x^{n}, y\right] & =m\left[x^{n}, y\right] y^{m-1} \\
& =m n x^{n-1}[x, y] y^{m-1} \\
& =m n x^{n-1} y^{m-1}[x, y] \\
& =n x^{n-1} m y^{m-1}[x, y] \\
& =n x^{m-1} x^{k}\left[x^{n}, y\right] \\
& =n x^{n+k-1}\left[x^{n}, y\right]
\end{aligned}
$$

Thus by using (2.16) and (2.17) we can write (2.15) as

$$
\begin{aligned}
n x^{n+k-1} x^{(n-1)(n+k-1)}\left[x^{n}, y\right] & =n x^{n+k-1}\left[x^{n}, y\right] . \\
n x^{n+k-1}\left(1-x^{(n-1)(n+k-1)}\right)\left[x^{n}, y\right] & =0 .
\end{aligned}
$$

Hence by using (2.18) we get,

$$
n x^{n+k-1}\left(1-x^{m d(n-1)(n+k-1)}\right)\left[x^{n}, y\right]=0 .
$$

Since $a \in D(R)$, we have

$$
a^{m d(n-1)(n+k-1)} \in Z(R) \cap D(R) \text { and } I a^{\operatorname{md}(n-1)(n+k-1)}=0 .
$$

By (2.19) we get

$$
n a^{n+k-1}\left(1-a^{m d(n-1)(n+k-1)}\right)\left[a^{n}, y\right]=0 .
$$

This can be written as

$$
\left(1-a^{m d(n-1)(n+k l-)}\right) n a^{n+k-1}\left[a^{n}, y\right]=0 .
$$

If $n a^{n+k-1}\left[a^{n}, y\right] \neq 0$. Then 


$$
1-a^{m(n-1)(n+k-1)} \in D(R)
$$

and $I\left(1-a^{m d(n-1)(n+k-1)}\right)=0$ and hence $I-0$, which is a contradiction. Thus we have

$$
n a^{n+k-1}\left[a^{n}, y\right]=0 \text {. }
$$

Now

$$
\begin{aligned}
m^{2} y^{m-1} y^{m-1}[a, y] & =m y^{m-1}[a, y] m y^{m-1}-a^{k}\left[a^{n}, y\right] m y^{m-1} \\
& =a^{k} m y^{m-1}\left[a^{n}, y\right]-a^{k} a^{n k}\left[\left(a^{n}\right)^{n}, y\right] \\
& =a^{m k+k} n\left(a^{n}\right)^{n-1}\left[a^{n}, y\right]=a^{m k+k} n a^{n-1} a^{(n-1)^{2}}\left[a^{n}, y\right] \\
& =a^{n k} a^{(n-1)^{2}} n a^{n+k-1}\left[a^{n}, y\right]-0 .
\end{aligned}
$$

Replacing $y$ by $y+1$ in (2.22) and using Lemma 3, we get

$$
m^{2}[a, y]-0 \text { for all } y \in R \text {. }
$$

Replacing $y$ by $x$, we get

$$
m^{2}[x, a]-0 \text { for all } x \in R \text {. }
$$

But $m^{2}$ and $n^{2}$ are relatively prime. Hence there exists integers $\alpha$ and $\beta$ such that $m^{2} \alpha+n^{2} \beta-1$. Multiplying (2.11) by $\beta$ and (2.23) by $\alpha$ and adding, we get

$$
[x, a]=0 \text { for all } x \in R \text {. }
$$

Hence $a \in Z(R)$, which proves our claim.

We know that $x^{n c}$ and $x^{m c m} \in Z(R)$. Thus

$$
\begin{aligned}
\left(x^{n c}-x^{n c m}\right) n x^{n+k-1}[x, y] & =n x^{n c} x^{n+k-1}[x, y]-n x^{n c m} x^{n+k-1}[x, y] \\
& =n x^{n+k-1}\left[x, x^{n c} y\right]-x^{n+m}\left[x, y^{m}\right] \\
& =n x^{n+k-1}\left[x, x^{n c} y\right]-\left[x,\left(x^{n c} y\right)^{m}\right] \\
& =n x^{n+k-1}\left[x, x^{n c} y\right]-n x^{n+k-1}\left[x, x^{m c} y\right]=0 .
\end{aligned}
$$

Thus $\left(x-x^{n c m-m c+1}\right) n x^{n+k-1} x^{m c-1}[x, y]=0$, i.e.

$$
n\left(x-x^{t}\right) x^{p}[x, y]-0 \text { for all } x, y \in R
$$

where $t=n c m-n c+1>1$ and $p=n+k+n c-2$.

We know that $y^{m d}$ and $y^{m \text { mh }} \in Z(R)$. Thus

$$
\begin{aligned}
\left(y^{m d}-y^{m d n}\right) m y^{m-1}[x, y] & =m y^{m d} y^{m-1}[x, y]-m y^{m t^{m}} y^{m-1}[x, y] \\
& =m y^{m-1}\left[x y^{m d}, y\right]-y^{m+} x^{k}\left[x^{m}, y\right] \\
& =m y^{m-1}\left[x y^{m d}, y\right]-x^{k}\left[\left(x y^{m d}\right)^{n}, y\right] \\
& =m y^{m-1}\left[x y^{m d}, y\right]-m y^{m-1}\left[x y^{m d}, y\right]-0
\end{aligned}
$$

Thus $m\left(y-y^{m d n-m d+1}\right) y^{m d-1} y^{m-1}[x, y]=0$. That is $m\left(y-y^{k}\right) y^{q}[x, y]=0$ for all $x, y \in R$, where $u=m d n-m d+1>1$ and $q=m d+m-2$. Interchanging $x$ and $y$, we get

$$
m\left(x-x^{4}\right) x^{q}[x, y]-0 \text { for all } x, y \in R \text {. }
$$

We know that $(m, n)=1$. Hence there exists integers $\alpha$ and $\beta$ such that $m \alpha+n \beta=1$. Multiplying (2.24) by $\beta\left(x-x^{4}\right) x^{q}$ and multiplying (2.25) by $\alpha\left(x-x^{t}\right) x^{p}$ and adding, we get

$$
\left(x-x^{\prime}\right)\left(x-x^{\prime \prime}\right) x^{p+q}[x, y]=0 \text { for all } x, y \in R
$$


This can be written as

$$
\left(x-x^{2} h(x)\right) x^{p+q+1}[x, y]=0 \text { for all } x, y \in R
$$

where $h(x)$ is a polynomial in $x$ with integers coefficients.

Suppose $R$ is not cummutative. Then by a well known result of Herstein [6], there exists $x \in R$ such that $x-x^{2} h(x) \notin Z(R)$. From this it is clear that $x \notin Z(R)$. Hence $x$ and $x-x^{2} h(x)$ is not a zero divisor. Hence $\left(x-x^{2} h(x)\right) x^{p+q+1}$ is also not a zero divisor. Thus

$$
[x, y]-0 \text { for all } y \in R
$$

This gives a contradiction. Hence $R$ is commutative.

CASE 2: Let $n>1$ and $m=1$. Then $\left({ }^{*}\right)$ can be written as

$$
x^{k}\left[x^{n}, y\right]=[x, y]
$$

Let $e=2^{k+n}-2>0$. Then

$$
\begin{aligned}
e[x, y] & =2^{k+n}[x, y]-2[x, y] \\
& =2^{k+n} x^{k}\left[x^{n}, y\right]-[2 x, y] \\
& =(2 x)^{k}\left[(2 x)^{n}, y\right]-[2 x, y] \\
& =[2 x, y]-[2 x, y]=0 .
\end{aligned}
$$

All commutators are central and hence by Lemma 2 ,

$$
\left[x^{e}, y\right]=e x^{e-1}[x, y]=0 \text { for all } x, y \in R \text {. }
$$

Hence $e^{e} \in Z(R)$. Now replacing $x$ by $x^{n}$ in (2.28) we get

$$
x^{n k}\left[\left(x^{n}\right)^{n}, y\right]=\left[x^{n}, y\right]
$$

Thus

$$
\begin{aligned}
x^{n k}\left[\left(x^{n}\right)^{n}, y\right] & =n x^{n k}\left(x^{n}\right)^{n-1}\left[x^{n}, y\right] \\
& =n x^{n k} x^{(n-1)} x^{(n-1)^{2}}\left[x^{n}, y\right] \\
& =n x^{n k-k} x^{n+k-1} x^{(n-1)^{2}}\left[x^{n}, y\right] \\
& =n x^{n+k-1} x^{(n-1)(n+k-1)}\left[x^{n}, y\right] \\
& =n x^{n-1} x^{(n-1)(n+k-1)} x^{k}\left[x^{n}, y\right] \\
& =n x^{n-1} x^{(n-1)(n+k-1)}[x, y] .
\end{aligned}
$$

and

$$
\left[x^{n}, y\right]=n x^{n-1}[x, y] .
$$

Thus, by using (2.30) and (2.31), we can write (2.29) as

$$
n x^{n-1} x^{(n-1)(n+k-1)}[x, y]=n x^{n-1}[x, y] .
$$

Thus

$$
n x^{n-1}\left(1-x^{(n-1)(n+k-1)}\right)[x, y]=0 .
$$

Thus, by using (2.32), we get

$$
n x^{n-1}\left(1-x^{e(n-1)(n+k-1)}\right)[x, y]=0
$$

Let $a \in D(R)$ then

$$
a^{e(n-1)(n+k-1)} \in Z(R) \cap D(R) \text { and } I a^{e(n-1)(n+k-1)}=0
$$


By using (2.33) we get

$$
n a^{n-1}\left(1-a^{e(n-1)(n+k-1)}\right)[a, y]=0 .
$$

Then

$$
\left(1-a^{e(n-1)(n+k-1)}\right) n a^{n-1}[a, y]=0 .
$$

If $n a^{n-1}[a, y] \neq 0$. Then

$$
\left(1-a^{e(n-1)(n+k-1)}\right) \in D(R)
$$

and $I\left(1-a^{e(n-1)(n+k-1)}\right)=0$. Hence $I=0$, which is a contradiction. Thus we have

$$
\left[a^{n}, y\right]=n a^{n-1}[a, y]=0 \text {. }
$$

Hence $a^{k}\left[a^{n}, y\right]=[a, y]=0$ for all $y \in R$. Now $a \in Z(R)$. We know that $x^{e}$ and $x^{e n} \in Z(R)$. Thus

$$
\begin{aligned}
\left(x^{e}-x^{e n+e k}\right)[x, y] & =x^{e}[x, y]-x^{e n+e k}[x, y] \\
& =\left[x^{e+1}, y\right]-x^{e n+e k} x^{k}\left[x^{n}, y\right] \\
& =\left[x^{e+1}, y\right]-x^{e k} x^{k}\left[\left(x^{e+1}\right)^{n}, y\right] \\
& =\left[x^{e+1}, y\right]-x^{(e+1) k}\left[\left(x^{e+1}\right)^{n}, y\right] \\
& =\left[x^{e+1}, y\right]-\left[x^{e+1}, y\right]=0 .
\end{aligned}
$$

Hence $\left(x-x^{e n+c k-e+1}\right) x^{e-1}[x, y]=0$. If $R$ is not commutative then by a well known result of Herstein [5] there exists $x \in R$ such that $x-x^{\nu} \notin Z(R)$ where $v=e n+e k-e+1>1$. By using smaller arguments as in the last paragraph of case 1 , we get a contradiction. Hence $R$ is commutative.

We give examples which show that all the hypotheses of our main theorem are essential. The following example show that $R$ is not cummutative if $m$ and $n$ are not relatively prime or the ring is without unity in the hypothesis of our main theorem.

EXAMPLE 1. Let

$$
R=\left\{\left(\begin{array}{lll}
0 & a & b \\
0 & 0 & c \\
0 & 0 & 0
\end{array}\right): a, b, c \in F, F: \text { field }\right\}
$$

Then $R$ is a ring without unity satisfying $x^{k}\left[x^{2}, y\right]-\left[x, y^{3}\right]$ and for all non-negative integer $k$. But $R$ is not commutative.

EXAMPLE 2. Let

$$
R=\left\{\left(\begin{array}{lll}
a & b & c \\
0 & a & d \\
0 & 0 & a
\end{array}\right): a, b, c, d \in G F(2)\right\}
$$

Then $R$ is a ring with unity satisfying $x^{k}\left[x^{4}, y\right]=\left[x, y^{4}\right]$ for all $x, y \in R$ and for all non-negative integer $k$. But $R$ is not commutative.

ACKNOWLEDGEMENT. I express my sincere thanks to the referee for his helpful suggestions.

\section{REFERENCES}

1. ASHRAF, M. and QUADRI, M. A., On commutatively of associative rings, Bull. Aust. Math. Sec. 38 (1988), 267-271.

2. BELL, H. E., On some commutativity theorems of Herstein, Arch. Math. 24 (1973), 34-38.

3. BELL, H. E., A commutativity study for periodic rings, Pacific J. Math. 70 (1977), 29-36. 
4. BELL, H. E., On the power map and ring commutativity, Canadian Math. Bull, 21 (1978), 399-404.

5. HERSTEIN, I. N., A generalization of a theorem of Jacobson, Amer. J. Math. 73 (1951), 756-762.

6. HERSTEIN, I. N., Two remarks on the cummutativity of rings, Canadian J. Math 7(1955), 411-412.

7. HERSTEIN, I. N., Power maps in rings, Michigan Math. J 8 (1961), 29-32.

8. JACOBSON, N., The structure of rings, A.M.S. Colloq. Publ. 37 (1964).

9. NICHOLSON, W. K. and YAQUB, A., A commutativity theorem for rings and groups, Canad. Math. Bull. 22 (1979), 412-413.

10. NICHOLSON, W. K. and YAQUB, A., A commutativity theorem, Algebra Universalis 10 (1980), 260-263.

11. PSOMOPOULOS, E., A commutativity theorem for rings, Math. Japon 29 (1984), 371-373.

12. PSOMOPOULOS, E., A commutativity theorem for rings and groups with constraints on commutators, Glasnik Mathematicki 20 (1985), 7-14. 


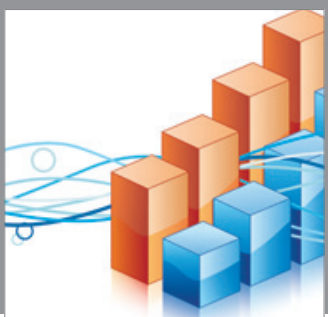

Advances in

Operations Research

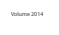

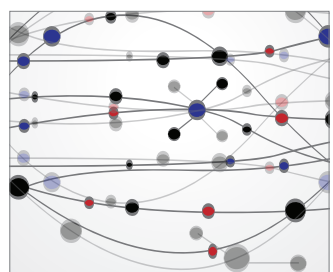

\section{The Scientific} World Journal
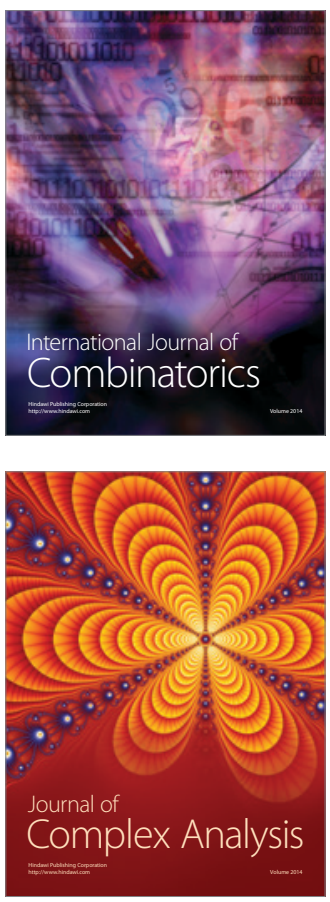

International Journal of

Mathematics and

Mathematical

Sciences
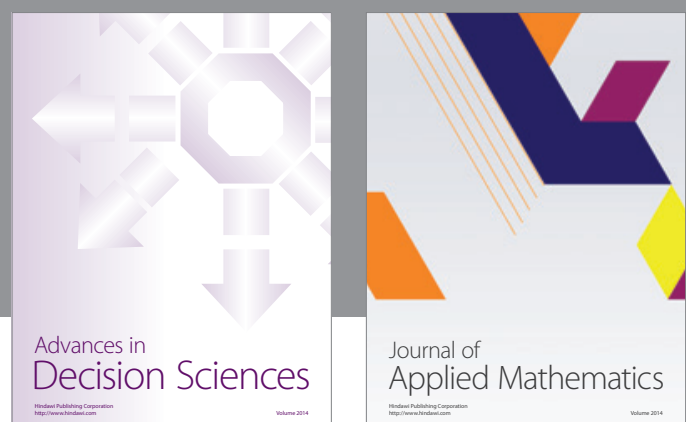

Journal of

Applied Mathematics
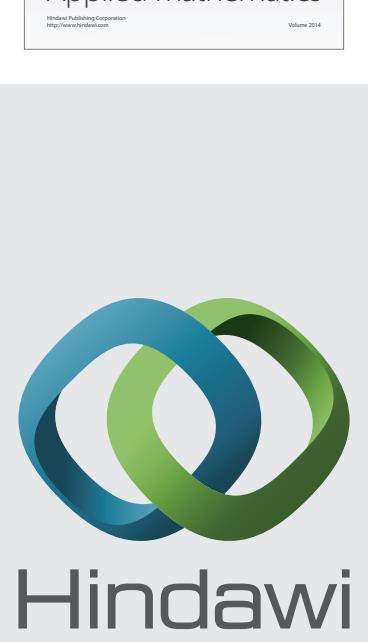

Submit your manuscripts at http://www.hindawi.com
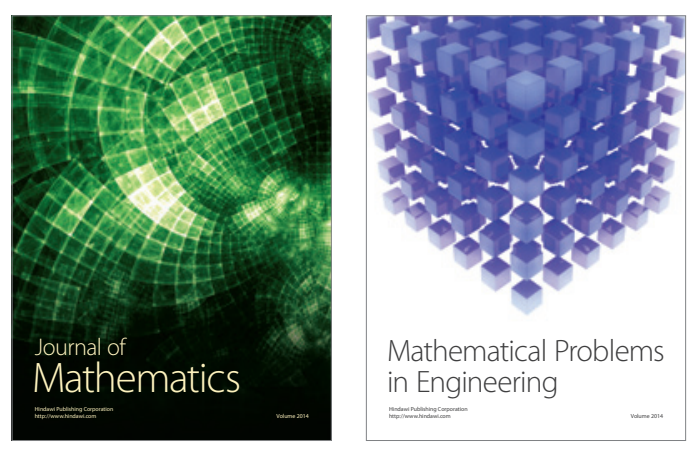

Mathematical Problems in Engineering
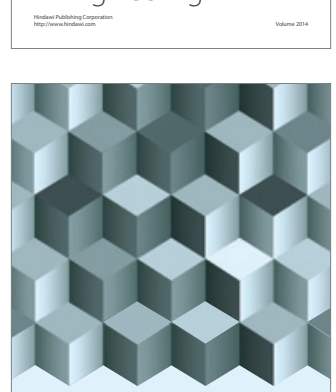

Journal of

Function Spaces
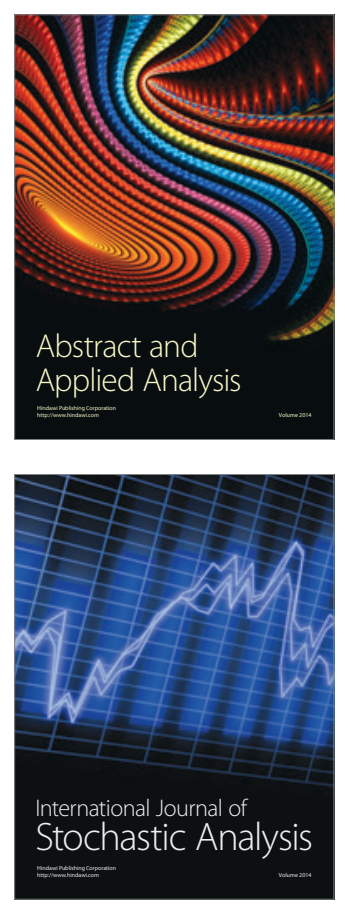

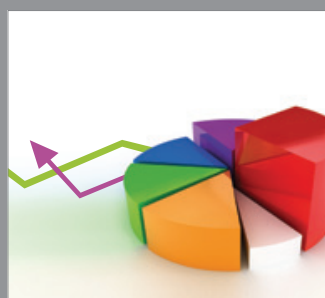

ournal of

Probability and Statistics

Promensencen
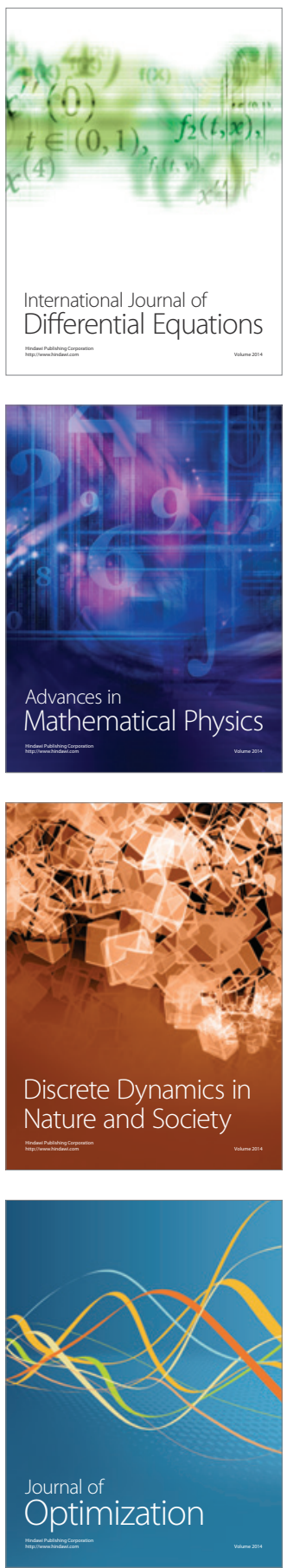